
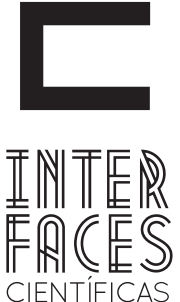

SAÚDE E AMBIENTE

ISSN IMPRESSO 2316-3313

ISSN ELETRÔNICO 2316-3798

DOI - 10.17564/2316-3798.2015v4n1p41-53

\title{
DEPRESSÃO NOS ACADÊMICOS DA ÁREA DE SAÚDE EM UMA FACULDADE PARTICULAR DE ARACAJU
}

Aline Melo Sentges Lima ${ }^{1}$

Sonia Lima
Erica Silva Barros ${ }^{2}$

\section{RESUMO}

Buscou-se analisar os diferentes graus da depressão nos cursos da área de saúde. 0 trabalho foi desenvolvido com os acadêmicos de Medicina, Enfermagem e Odontologia em uma universidade particular de Aracaju, matriculados em 2013. Aplicou-se o Inventário de Depressão de Beck em 383 participantes. A distribuição dos participantes por curso mostrou que $11,62 \%(n=44)$ cursavam Medicina; 24,50\% ( $n=94)$ Odontologia e $63,87 \%(n=245)$ Enfermagem. $71,27 \%$ eram do sexo feminino e $28,72 \%$ do masculino. A faixa etária de 26 a 33 anos representou 55,61\%. No curso de Medicina não houve depressão em 36 indivíduos, leve em cinco e moderada em três. 0 curso de Odontologia apresentou ausência de depressão em 40 estu- dantes, leve em 33, moderada em 16 e grave em cinco. No curso de Enfermagem verificou-se ausência de depressão em 78, leve em 98, moderada em 48 e grave em 21. Assim, a depressão em estudantes da área de saúde é um fator de risco que justifica medidas preventivas. Diante disso, foi sugerido aos orientadores dos referidos cursos utilizar medidas preventivas de apoio emocional, visando à saúde mental dos futuros profissionais.

\section{PALAVRAS-CHAVE}

Depressão. Saúde Mental. Estudantes de Ciências da Saúde. 


\section{ABSTRACT}

Was sought to analyze the different degrees of depression in health courses. The study was conducted with medical students, nursing and dentistry in a private university in Aracaju, enrolled in 2013. We used the Beck Depression Inventory in 383 participants. The distribution of participants per course showed that $11.62 \%(n=44)$ were enrolled medicine; $24.50 \%(n=94)$ Dentistry and $63.87 \%(n=$ 245) nursing. $71.27 \%$ were female and $28.72 \%$ male. The age group $26-33$ years was $55.61 \%$. In medical school there was no depression in 36 individuals, mild and moderate in 5 to 3 . The course of dentistry showed no depression in 40 students, mild in

\section{RESUMEN}

Se trató de analizar los diferentes grados de depresión en los cursos de salud. El estudio se realizó con estudiantes de medicina, enfermería y odontología en una universidad privada de Aracaju, registrada en 2013. Se utilizó el Inventario de Depresión de Beck en 383 participantes. La distribución de participantes por curso mostró que $11,62 \%(n=44)$ se inscribieron en la medicina; $24,50 \%(n=94)$ Odontología y $63,87 \%(n=245)$ Enfermería. $71,27 \%$ eran hombres y $28,72 \%$ mujeres. El grupo de edad 26 a 33 años de edad representaron el 55,61\%. En la escuela de medicina no había depresión en 36 individuos, tomar los días 5 y moderada supuesto 3 . Odontología presentó ausencia de depresión por 40
33, moderate in 16 and severe in 5 . In the course of nursing there was no depression in 78 , mild in 98 , moderate in 48 and severe in 21 . So the depression in healthcare students is a risk factor that justifies preventive measures.Therefore, it was suggested to the guiding of these courses use preventive measures of emotional support aimed at mental health of future professionals.

\section{KEYWORDS}

Depression. Mental Health. Students. Health Occupations. estudiantes, leve en 33, moderada en 16 y severa en 5. Curso de Enfermería fue encontrado ausencia de la depresión en el 78, leve en 98 , moderada en 48 y severa en 21 . Así que la depresión en estudiantes de la salud es un factor de riesgo que justifique las medidas preventivas. Por lo tanto, se sugirió que el guiado de estos cursos usar medidas preventivas de apoyo emocional, dirigidos a la salud mental de los futuros profesionales.

\section{PALABRAS CLAVE}

Depresión. Salud Mental. Los Estudiantes. Ciencias de La Salud. 


\section{INTRODUÇÃO}

A depressão é um dos transtornos do humor, caracterizada por lentificação dos processos psíquicos, humor depressivo e/ou irritável, redução da energia com desânimo e cansaço fácil, incapacidade parcial ou total de sentir alegria e/ou prazer, desinteresse, apatia ou agitação psicomotora. Além da dificuldade de concentração, pensamentos de cunho negativo, com perda da capacidade de planejar o futuro e alteração do juízo da realidade (COUTINHO, GONTIÈS \& SÁ; 2003). Os sintomas depressivos podem ter duração variada, com episódios únicos, recorrentes, crônicos ou breves e com intensidades diferentes, comprometendo as atividades cotidianas de forma leve, moderada ou grave (LIMA, 2004).

A depressão aparece como um agravo que vem alterar o sistema normal de regulação do humor, afetando as respostas emocionais do organismo. Nesse contexto, é a tristeza que caracteriza essa doença. Os outros sintomas são consequências da alteração fisiológica que estão na origem deste desregulamento (Costa ET AL., 2012).

Atualmente, a depressão é considerada um problema de saúde mundial tão frequente quanto diabetes e hipertensão. Estima-se que $20 \%$ da população geral sofrem deste transtorno, ou seja, a cada quatro pessoas uma irá fazer tratamento para esse agravo, pelo menos alguma vez na vida. No Brasil, aproximadamente 24 a 30 milhões de pessoas apresentam, apresentaram ou virão a ter pelo menos um episódio depressivo ao longo da vida. Indivíduos de ambos os sexos, de todas as etnias, idades e condições sociais podem ser acometidos, tendo nas mulheres o grupo mais vulnerável, na proporção de duas a três para cada homem (GOMES-OLIVEIRA ET AL., 2012).

Pesquisas realizadas com acadêmicos da área de saúde vêm registrando um índice elevado de casos de depressão, com predominância nas mulheres (97\%) com idade média de 20 anos (CUNHA; 2012). Tradicionalmente, os cursos da área de saúde são aceitos como difíceis e/ou trabalhosos, por exigir dos alunos: dedica- ção, esforço, sacrifício e, sobretudo, resistência física e emocional. A associação entre estudar na área de saúde e estar em condição crônica de estresse é mundialmente conhecida (HAGHPARAST ET AL., 2012).

A área de saúde é uma carreira que exige algumas características e expõe seus aspirantes a numerosas situações de estresse que muitos não têm condições de enfrentar, por características pessoais, falta de preparo ou por motivos e situações diversas, inclusive inerentes ao próprio curso. A vocação em ser um profissional da área de saúde é uma questão que tem despertado o interesse de alguns estudiosos em todo o mundo, havendo uma discussão sobre a necessidade de ampliar os critérios de seleção dos candidatos aos cursos, incluindo, além das aptidões físicas, sensoriais ou intelectuais, aquelas expressas por respeito, moral, bom senso, objetividade, responsabilidade e resiliência (GOMES-OLIVEIRA ET AL., 2012).

$O$ processo de exaustão emocional surge naqueles que não conseguem superar as adversidades, caracterizando-se pelas sensações de não poder dar mais de si mesmo e haver chegado ao próprio limite. Os indivíduos se tornam ansiosos, estudam mais e mais, dormem pouco, têm pouco tempo livre, perdem oportunidades de ter relações sociais e recreações, tornando-se mais vulneráveis aos distúrbios mentais (FIORANO, 2002).

$\mathrm{Na}$ tentativa de distanciamento, de minimizar a exaustão, começam a tratar os demais com indiferença, e impessoalidade, adotando atitudes irônicas ou cínicas, caracterizando o que vem sendo chamado de desumanização. Essa questão tem sido bastante enfatizada nos anos recentes, devido à observação da acentuação de atitudes céticas e da diminuição dos sentimentos humanísticos em muitos estudantes (FIORANO, 2002).

As faculdades da área de saúde são ambientes hostis, de muita competição. Três fases psicológicas enfrentadas por estes acadêmicos devem ser destacadas: (1) euforia inicial, na qual são ativadas crenças de caráter onipotente; (2) decepção, causada pela extrema mudança de hábitos do cotidiano e, às vezes, pelo desempenho insatisfatório nas discipli- 
nas; (3) estágio, composto por um período de adaptação e, ao mesmo tempo, por uma alta competitividade pela especialização.

Além disso, o medo de falhar ou até frustrações quanto à realização profissional e falta de reconhecimento, são sentimentos comuns, que não sendo bem administrados, podem trazer consequências ruins à saúde do profissional, já que, frente ao erro, este pode ser tomado por sentimento de culpa pelo fracasso e aparente impotência (FUREGATO ET AL., 2006).

Os profissionais da área de saúde constituem uma população de risco para vários distúrbios de comportamento, crises e tentativa de suicídio. Esse fato é comprovado pelo número crescente de acadêmicos e profissionais que optam pela interrupção de suas carreiras e, às vezes, da própria vida, por causa de transtornos psíquicos. O conhecimento dos problemas que afligem os estudantes da área de saúde é de fundamental importância na execução de planejamento adequado para atender às suas solicitações e, também, para fornecer subsídios à instituição de ensino, com o intuito de auxiliá-la no aprimoramento da formação discente e de suas relações interpessoais no ambiente universitário (GOMES-OLIVEIRA ET AL., 2012).

Os estudantes da área de saúde estão se preparando para lidar com vidas, e a sua condição psicológica influencia na sua formação e futura atuação. Portanto objetivou-se identificar a prevalência de depressão nos acadêmicos de Medicina, Enfermagem e Odontologia em uma universidade particular de Aracaju-Sergipe.

\section{MÉTODOS}

Estudo quantitativo aleatório com acadêmicos de Medicina, Enfermagem e Odontologia de uma universidade particular de Aracaju-Sergipe, mediante questionários autoaplicáveis.

A população total de estudantes foi de 2306 . Distribuída em 268 de Medicina, 1473 de Enfermagem e 565 de Odontologia, matriculados em uma universidade particular de Aracaju-Sergipe no ano de 2013 e 2014. Baseado em estudo prévio de Coutinho e Salda- nha (2012), que estimaram a prevalência populacional de depressão em $20 \%$, calculou-se uma amostra probabilística, fundamentada na fórmula discriminada abaixo, considerando um erro de $5 \%$ e uma precisão de $1 \%$, resultando numa amostragem de 384 participantes (Tabela 1).

De acordo com a porcentagem amostral proporcional à quantidade de alunos matriculados em cada curso; $11,62 \%$ são de Medicina resultando em 44 participantes; $24,50 \%$ são de Odontologia resultando em 94 participantes e $63,87 \%$ são de Enfermagem resultando 245 participantes.

Tabela 1 - Parâmetros adotados para o cálculo amostral

\begin{tabular}{|l|l|l|}
\hline Tamanho da população & N & 2306 \\
\hline Erro alfa & A & 0,05 \\
\hline Nível de Confiança & 1-a & 0,95 \\
\hline Z de (1-a) & Z(1-a) & 1,96 \\
\hline Prevalência de depressão & P & 0,20 \\
\hline Complemento de p & Q & 0,97 \\
\hline Precisão & D & 0,01 \\
\hline Tamanho da amostra & N & 383 \\
\hline
\end{tabular}

Fonte: Dados da pesquisa.

Foi redigido um Termo de Consentimento Livre e Esclarecido, conforme as normas do Conselho Nacional de Saúde do Ministério da Saúde, explicitadas na resolução 466/12. Foram esclarecidos aos estudantes de Medicina, Enfermagem e Odontologia que os mesmos poderiam se recusar a participar do estudo, interrompê-lo a qualquer momento, não responder a alguma pergunta do questionário e que os dados obtidos seriam sigilosos. A pesquisa não envolveria qualquer tipo de intervenção programada com os participantes. Na recusa de participar do estudo, os acadêmicos foram substituídos a partir de uma nova escolha aleatória com novo sorteio.

O questionário autoaplicável utilizado foi o Inventário de Depressão de Beck - Beck DepressionInventory 
(BDI) - que trabalha com uma amplitude de itens para avaliar depressão, usado tanto em pesquisa como na prática clínica.O BDI foi traduzido para vários idiomas e validado em diferentes países. Em 1996, o inventário foi novamente avaliado para incluir os critérios do DSM-IV, com o objetivo de acrescentar um episódio depressivo maior (BECK; STEER e CARBIN, 1988).

A escala original consiste de 21 itens, incluindo sintomas e atitudes, cuja intensidade varia de zero a três. Os itens referem-se à tristeza, pessimismo, sensação de fracasso, falta de satisfação, sensação de culpa, sensação de punição, autodepreciação, autoacusações, ideias suicidas, crises de choro, irritabilidade, retração social, indecisão, distorção da imagem corporal, inibição para o trabalho, distúrbio do sono, fadiga, perda de apetite, perda de peso, preocupação somática e diminuição de libido.

Há várias propostas de diferentes pontos de corte para distinguir os níveis de depressão, utilizando o BDI. De acordo com Beck; Steer e Carbin (1988), a escolha do ponto de corte adequado depende da natureza da amostra e dos objetivos do estudo. Para amostras de pacientes com transtorno de humor o Center for Cognitive Therapy recomenda os seguintes pontos de corte: menor que 10 = sem depressão ou depressão mínima; de 10 a 18 = depressão, de leve a moderada; de 19 a 29 = depressão, de moderada a grave; de 30 a 63 = depressão grave (BECK; STEER; CARBIN, 1988).

A aplicação do BDI foi em dias e horários nos quais as turmas estavam reunidas para alguma atividade em sala de aula. Os professores, responsáveis pelas turmas, foram informados previamente para que cedessem os 30 minutos iniciais de sua aula.

Antes de distribuir o instrumento, as duas acadêmicas do curso de Medicina vinculadas à pesquisa esclareceram os objetivos da mesma, a importância da colaboração ao ensino e à pesquisa do tema, bem como a identificação e o encaminhamento dos casos identificados entre eles. Ressaltou-se a importância do silêncio e da concentração para a garantia de melhores resultados, de não ser deixada nenhuma questão sem resposta e que, em caso de dúvida, uma das duas acadêmicas presentes deveria ser requisitada. Foram informados sobre os procedimentos para o preenchimento do inventário, de que os alunos detectados com depressão seriam individualmente procurados e orientados, e que a atividade era uma tarefa simples, sem prejuízos nem custos, garantindo o anonimato.

O critério de inclusão consistiu na aceitação do estudante dos cursos de Medicina, Enfermagem e Odontologia em participar da pesquisa após ter lido e assinado o Termo de Consentimento Livre e Esclarecido. O critério para exclusão consistiu daqueles que apresentarem doença crônica que sabidamente predispõe à presença de depressão, como vitiligo, hipotireoidismo, diabetes melitus e esclerose lateral amiotrófica.

O curso de Medicina na instituição avaliada era da metodologia Problem Based Learning (PBL), e o curso de odontologia e enfermagem eram da metodologia tradicional.

O trabalho foi aprovado pelo Comitê de Ética em Pesquisa com Seres Humanos da Universidade Tiradentes - SE, com permissão dos departamentos de medicina, enfermagem e odontologia da Universidade Tiradentes aos requisitos da portaria do CNS 466/12 no cadastro $040613 R$.

Os dados foram analisados de forma descritiva e analítica. As variáveis numéricas foram observadas quanto à distribuição de normalidade por meio do teste de Shapiro-Wilk. Nos casos em que os pressupostos foram atendidos, as mesmas foram apresentadas por meio de média e desvio padrão (DP), caso contrário por meio de mediana $(\mathrm{Md})$ e seus quartis $\left(1^{0}-3^{\circ}\right)$. As variáveis categóricas foram apresentadas por meio de frequência absoluta e relativa. A prevalência foi calculada por meio do número de indivíduos com o desfecho (de leve a grave) dividido pelo número total de indivíduos na amostra, com seus respectivos intervalos de confiança de $95 \%$.

No modelo univariado, para a análise das associações entre a variável dependente (depressão) com as variáveis independentes (faixa etária, gênero e curso), foi aplicado o teste do qui-quadrado $\left({ }^{2}\right)$ com correção de Yatesou Exato de Fisher. Com o objetivo de analisar a magnitude dessas associações, foi realizada a análise multivariada por meio da regressão de Poisson com 
ajuste robusto de variância representada por valores de razão de prevalência (RP) e intervalo de confiança de $95 \%$ [IC95\%]. Para este modelo, foram consideradas as variáveis que tiveram um valor de $\mathrm{P}<0,20$ na análise univariada.

No modelo ajustado da regressão de Poisson, todas as variáveis independentes foram inseridas simultaneamente. A significância estatística foi adotada em 5\% $(P \leq 0,05)$. Os programas estatísticos utilizados foram o Statistical Package for the Social Sciences (SPSS 15.0) e o Stata/SE (SANTOS ET AL.; 2003).

\section{RESULTADOS}

Fizeram parte do estudo 383 acadêmicos, sendo $273(71,3 \%)$ mulheres com mediana de 28 anos de idade $(23-30,5)$. Dos 110 participantes do gênero masculino (28,7\%), a média foi também de 28 anos de idade (22-31). Não houve diferença na idade entre os gêneros $(p=0,875)$. Ao serem comparadas as idades entre os gêneros em cada curso, apenas no de Odontologia foi encontrada diferença entre homens e mulheres $(p=0,023)$. A faixa etária e o gênero dos acadêmicos de cada curso são apresentadas na Tabela 2.

Tabela 2 - Distribuição das proporções da classificação das pontuações do QSF de acordo com a situação conjugal, Aracaju, 2015

\begin{tabular}{|c|c|c|c|c|c|c|c|c|c|c|}
\hline & \multicolumn{3}{|c|}{ Sem Companheiro } & \multicolumn{3}{|c|}{ Com companheiro } & \multicolumn{2}{|c|}{ Ambos } & \multirow[b]{2}{*}{$\%$ a } & \multirow{2}{*}{$\mathrm{p}$} \\
\hline & $\mathrm{n}$ & $\%$ & $\% a$ & $\mathrm{n}$ & $\%$ & $\% a$ & $\mathrm{~N}$ & $\%$ & & \\
\hline Nulo/ruim & 12 & 15,0 & 15,0 & 10 & 12,5 & 12,5 & 22 & 27,4 & 27,4 & \\
\hline Ruim/desfavorável & 4 & 5,0 & 20,0 & 4 & 5,0 & 17,5 & 8 & 10,0 & 37,4 & \\
\hline Desfavorável/regular & 16 & 20,0 & 40,0 & 15 & 18,7 & 36,2 & 31 & 38,8 & 76,2 & 0,22 \\
\hline Regular/bom & 8 & 10,0 & 50,0 & 8 & 10,0 & 46,2 & 16 & 20,0 & 90,2 & \\
\hline Bom/excelente & 2 & 2,5 & 52,5 & 1 & 1,3 & 47,5 & 3 & 3,8 & 100,0 & \\
\hline
\end{tabular}

$\%$ Frequéncia relativa acumulada.

Fonte: Dados da pesquisa. 
A prevalência de depressão entre os acadêmicos foi de $62,92 \%[$ IC95\%= 57,98-67,61]. Houve diferença significativa entre os cursos, sendo maior no de Enfermagem com 71,02\% [IC95\%= $65,05-76,34](p=0,014)$, seguido por Odontologia com $60,64 \%$ [IC95\%=50,53-69,91] e em menor percentual no curso de Medicina, com22,73\%
[IC95\%= 12,84-36,99] de estudantes com depressão. Em relação aos graus de depressão (leve, moderada ou grave), foi também verificada associação significativamente maior nos acadêmicos do curso de Enfermagem. Desde que do total de 241 acadêmicos com depressão, 174 (72,2\%) eram deste curso (Tabela 3).

Tabela 3 - Distribuição das proporções da classificação das pontuações do QSF de acordo com o nível socioeconômico, Aracaju, 2015

\begin{tabular}{|c|c|c|c|c|c|c|c|c|c|c|}
\hline & \multicolumn{3}{|c|}{ Baixo } & \multicolumn{3}{|c|}{ Médio/baixo } & \multicolumn{3}{|c|}{ Médio } & \multirow[t]{2}{*}{ p } \\
\hline & $\mathrm{n}$ & $\%$ & $\%^{a}$ & $\mathrm{n}$ & $\%$ & $\%^{\mathrm{a}}$ & $\mathrm{N}$ & $\%$ & $\%^{\mathrm{a}}$ & \\
\hline nulo/ruim & 10 & 12,5 & 12,5 & 5 & 6,1 & 6,1 & 7 & 8,8 & 8,8 & \\
\hline ruim/desfavorável & 3 & 3,8 & 16,3 & 4 & 5,0 & 11,1 & 1 & 1,3 & 10,1 & \\
\hline desfavorável/ regular & 7 & 8,8 & 25,1 & 4 & 5,0 & 16,1 & 20 & 25,0 & 35,1 & \\
\hline regular/bom & 2 & 2,5 & 27,6 & 1 & 1,2 & 17,3 & 13 & 16,2 & 51,3 & \\
\hline bom/ excelente & 3 & 3,8 & 31,4 & 0 & 0 & 0 & 0 & 0 & 0 & \\
\hline Total & 25 & 31,4 & & 14 & 17,3 & & 41 & 51,3 & & \\
\hline
\end{tabular}

$\%^{a}$ Frequência relativa acumulada; *Associação estatisticamente significativa a nível de 0,001, exato de Fisher Fonte: Dados da pesquisa. 
Apesar de o gênero feminino apresentar uma frequência maior de casos de depressão, não houve associação entre as variáveis graus de depressão e gênero. Ao avaliar a depressão entre os gêneros em cada curso, também não foi encontrada associação entre os cursos de Medicina ( $p=$ $0,818)$, Enfermagem $(p=0,245)$ e Odontologia $(p=$ 0,505) (Tabela 4).

Tabela 4 - Distribuição da frequência absoluta e relativa dos graus de depressão do IDB e os gêneros dos acadêmicos de enfermagem, medicina e odontologia

\begin{tabular}{|c|c|c|c|c|c|}
\hline & \multicolumn{2}{|c|}{$\begin{array}{l}\text { Feminino } \\
(\mathrm{n}=273)\end{array}$} & \multicolumn{2}{|c|}{$\begin{array}{l}\text { Masculino } \\
(n=110)\end{array}$} & \multirow[t]{2}{*}{$\mathrm{p}$} \\
\hline & $\mathrm{n}$ & $\%$ & $\mathrm{n}$ & $\%$ & \\
\hline Ausência de depressão & 100 & 70,4 & 42 & 29,6 & \\
\hline Depressão leve a moderada & 78 & 75,7 & 25 & 24,3 & 0,937 \\
\hline Depressão moderada a grave & 75 & 67 & 37 & 33 & \\
\hline Depressão grave & 20 & 76,9 & 6 & 23,1 & \\
\hline
\end{tabular}

Fonte: Dados da pesquisa.

Ao verificar a presença de associação entre depressão e faixas etárias, foi identificada associação significante $(p=0,001)$, entre os acadêmicos de 26 a 33 anos, pois do total de 241 acadêmicos com depressão, $153(63,49 \%)$ eram dessa faixa etária (Tabela 5).

Tabela 5 - Distribuição das frequências absolutas e relativas dos graus de depressão do IDB e as faixas etárias dos acadêmicos de enfermagem, medicina e odontologia

\begin{tabular}{llllllll}
\hline & $\begin{array}{l}18 \text { a 25 anos } \\
(\mathrm{n}=121)\end{array}$ & \multicolumn{2}{l}{$\begin{array}{l}26 \text { a 33 anos } \\
(\mathrm{n}=218)\end{array}$} & \multicolumn{2}{l}{$\begin{array}{l}34 \text { a } 40 \text { anos } \\
(\mathrm{n}=44)\end{array}$} & $\mathrm{p}$ \\
\cline { 2 - 9 } & $\mathrm{n}$ & $\%$ & $\mathrm{n}$ & $\%$ & $\mathrm{n}$ & $\%$ & \\
Ausência de depressão & 65 & 45,8 & 65 & 45,8 & 12 & 8,5 & \\
Depressão leve a moderada & 23 & 22,3 & 64 & 62,1 & 16 & 15,5 & $0,001^{*}$ \\
Depressão moderada a grave & 28 & 25 & 71 & 63,4 & 13 & 11,6 & \\
Depressão grave & 5 & 19,2 & 18 & 69,2 & 3 & 11,5 & \\
\hline
\end{tabular}

*Presença de associação $(P \leq 0,05)$.

Fonte: Dados da pesquisa. 
Ao avaliar a depressão entre as diferentes faixas etárias em cada curso, apenas no curso de Enfermagem houve associação $(p=0,031)$. Neste, de um total de 174 acadêmicos com depressão, 115 (66,09\%) eram de uma faixa etária entre 26 a 33 anos. Não foi encontrada associação no curso de Medicina ( $p=$ $0,095)$ e Odontologia $(p=0,613)$.

\section{DISCUSSÃO}

Nos últimos anos, a saúde mental dos estudantes universitários tornou-se foco de atenção não só dos especialistas da área de saúde, mas da sociedade em geral (SANTOS ET AL., 2003). Os acadêmicos da área de saúde são submetidos a grande carga horária de estudo, bem como a imposição de um alto nível de cobrança, não só pela sociedade e instituição de ensino, bem como pelo próprio indivíduo. Estes também ficam expostos ao contato estreito com portadores de diferentes doenças e prognósticos ruins, sem um amadurecimento emocional nem um preparo psicológico para lidar com tais situações que enfrentam com os pacientes, o que muitas vezes podem favorecer a episódios de depressão (NARDI, 2000).

Observou-se que a incidência de depressão nos acadêmicos da área de saúde, em geral, foi de 56\%; a partir da análise amostral em faculdades distintas nos Estados Unidos (RIMMER; HALIKAS e SCHUCKIT, 1982). Avaliando uma faculdade de cada região do Brasil, evidenciou que a incidência geral de sintomas depressivos nos acadêmicos dessa área foi de $63 \%$, com maior prevalência na região sudeste e sul (OHL, 1991).

No presente estudo a prevalência de depressão, em estudantes de Enfermagem, Odontologia e Medicina de Sergipe, foi de $62,92 \%$, mostrando uma alta incidência desta afecção, corroborando os achados dos estudos citados, o que justifica um investimento na avaliação e monitoramento psicológico dos acadêmicos.

Em uma pesquisa com duas turmas de Enfermagem na cidade de São Paulo, no período diurno, com 65 alunos e noturno com 49 alunos. $O$ instrumento de avaliação foi o Inventário de Depressão de Beck (IDB). Com base nas respostas do IDB foi verificado que $15,4 \%$ dos alunos do período diurno e $28,6 \%$ do noturno tinham sinais indicativos de depressão. Destes, vinte alunos (14\%) podiam ser classificados com depressão moderada e nove com depressão grave (FIORANO; 2002).

Kawakame e Gracia (2000), analisando as experiências clínicas iniciais de estudantes brasileiros na graduação em Enfermagem, desde o primeiro período até sua conclusão, observou que há altos índices de depressão (24\%). Estes índices surgiram e foram aumentando com o decorrer do curso e diante das decepções com situações da prática nos estágios supervisionados. Os acadêmicos do curso de Enfermagem de Sergipe apresentaram os maiores índices de sintomas depressivos, quando comparados ao curso de Odontologia e de Medicina $(p=0,014)$. No total de 241 acadêmicos com depressão, 174 (72,2\%) eram do curso de Enfermagem, podendo levantar como hipóteses uma provável decepção diante da expectativa com o curso e/ou desgaste pela tensão no ambiente hospitalar.

Newbury-Birch; Lowry e Kamali (2002), em análise da depressão com estudantes da área de Odontologia em Londres, verificaram a presença de sintomas de ansiedade e nervosismo que evoluíam em $11 \%$ dos casos para depressão, comprometendo 0 rendimento acadêmico.

Já no Brasil, segundo Aguiar (2007), 73,6\% dos estudantes de Odontologia apontam que as suas atividades na faculdade são fonte de estresse, evidenciando uma prevalência de $28,3 \%$ de depressão. Nesta pesquisa os acadêmicos de Odontologia apresentaram o segundo maior índice de depressão, com prevalência de $60,64 \%(p=0,002)$. Estes estudantes lidam com a prática clínica precoce na sua atividade acadêmica, 0 que pode gerar insegurança, justificando a prevalência de depressão encontrada nesta pesquisa.

Moro, Valle e Lima (2005) revelaram uma prevalência de $26,8 \%$ de casos depressivos nos estudantes de Medicina da universidade da região de Joinville (SC). Curso de metodologia tradicional, mostrou uma incidência de $15 \%$ na transição das disciplinas básicas para as clínicas. Lima, Domingues e Cerqueira (2006) realizaram um estudo transversal 
com 551 universitários de um curso de Medicina de Botucatu (SP) de metodologia Problem Based Learning (PBL). Este autor verificou uma prevalência de transtornos mentais de $44,7 \%$ associando-se a dificuldade para fazer amigos, avaliação ruim sobre 0 desempenho escolar, pensamento de abandono do curso e falta de apoio emocional.

Costa e outros autores (2012) realizaram um estudo com os últimos dois anos, fase do internato, do curso de Medicina da Universidade Federal de Sergipe, que utiliza a metodologia tradicional, verificando $40,5 \%$ de casos de depressão, com sintomas que variaram de leve a moderado. A depressão ocorreu em maior porcentagem nos estudantes que já pensaram em abandonar o curso, naqueles com desempenho regular, nos que se sentiam incapazes de serem bons médicos até aquele momento e nos que se sentiam com alterações emocionais.

Esses autores sugeriram que, além das etapas trabalhosas do curso de Medicina, deve haver características individuais que podem determinar os sintomas depressivos. Na presente pesquisa, realizada também em Sergipe, com estudantes de Medicina submetidos à metodologia $\mathrm{PBL}$, apresentaram prevalência de depressão 22,73\%, com ausência de depressão grave, sendo significativamente menor quando comparada aos cursos de Enfermagem e Odontologia de cursos tradicionais da mesma universidade.

Moore (1997) constatou a redução dos sintomas de ansiedade e depressão em $12 \%$ dos acadêmicos na Escola de Medicina de Harvard, ao aderir completamente à metodologia PBL. Este modelo de ensino foi iniciado nessa escola, em 1984, implantado como programa curricular paralelo ao currículo tradicional, voluntário para estudantes de Medicina e para professores. Passados os primeiros anos e após uma avaliação comparativa entre o processo educacional tradicional e o novo currículo, Harvard passou a desenvolver um currículo único que tinha como estratégias o processo ensino-aprendizagem, centrado no estudante e o resgate da segurança do acadêmico, diante da relação médico-paciente como elemento agregador de conteúdos biopsicossociais.
O PBL foi inicialmente desenvolvido na Universidade de Mc Master, no Canadá, no final da década de 1960, por um grupo de docentes que pretendia criar um programa para o curso de Medicina centrado no aluno. 0 foco deste novo método de ensino é desenvolver competências gerais como: atenção à saúde, tomada de decisões, comunicação, liderança, administração e gerenciamento, visando a formação de um profissional ativo na sociedade (NARDI, 2000).

As características deste método são a organização temática em torno de problemas, a integração interdisciplinar, imbricando componentes básicos e clínicos e a ênfase no desenvolvimento cognitivo, além do aprendizado contextual, com uma visão holística do problema. Haghparast (2007) afirma que um dos principais objetivos desse método é ensinar o aluno a aprender, a fim de que busque o conhecimento, se sentindo capaz de manter uma postura de estudo e aprimoramento depois de formado, garantindo-lhe a atualização profissional, tão necessária no mundo globalizado.

Desta forma, o método PBL proporciona ao acadêmico a capacidade para resolução dos problemas, o que reduz o estresse e promove segurança na atuação profissional. Existem, assim, perspectivas positivas e promissoras em relação ao ensino da prática médica com foco no binômio ensino-aprendizagem por meio de metodologias ativas, problematização em cenários diversos, vivência comunitária, relações interdisciplinares e multiprofissionais (NARDI, 2000).

Os rumos das Escolas Médicas no Brasil e as propostas de mudanças curriculares, que se voltam à promoção de metodologias de ensino centradas no aluno, promovendo redução de sintomas depressivos. Entretanto, tendem a desenvolver experiências copiadas com modelos de outras nações, sem reflexão crítica, não conseguindo problematizar a organização do currículo no contexto da sociedade contemporânea brasileira. Aguiar (2007) relata a necessidade de uma melhor adequação dessas novas metodologias no Brasil visando a saúde mental dos estudantes.

Em épocas diferentes, a presença de sintomas depressivos foi evidenciando tanto na metodologia PBL como na tradicional. Eis que a mudança recente para 
a metodologia PBL promove um monitoramento constante do processo ensino aprendizagem do aluno, possibilitando uma atenção precoce ao surgimento de sintomas depressivos, ansiedade e estresse. Independente da metodologia é necessário o diagnóstico precoce de depressão nesses acadêmicos, visando a criação de estratégias defensivas adequadas para ajudá-los a enfrentar os problemas inerentes à área que escolheram.

Furegato (2006) afirma maior prevalência de depressão em mulheres associada ao curso de Enfermagem. Aguiar (2007) no curso de Medicina e Odontologia verificou que os índices depressivos nos homens ocorreram em 54,8\% na amostra avaliada de188 estudantes, com 135 de Medicina e 53 de Odontologia. Na presente pesquisa o gênero feminino apresentou a maior frequência de casos de depressão nos três cursos avaliados. Embora seja sabido ser maior a predisposição de depressão no gênero feminino (2004), não dever ser negligenciada a possibilidade de sintomas depressivos no gênero masculino.

Coutinho (2003) analisa que o transtorno depressivo possa afetar as pessoas em qualquer idade, indicando que os sintomas são altamente prevalentes principalmente nas fases tardias da vida, em todo o mundo. No presente estudo houve associação significativa de depressão e idades mais elevada entre os acadêmicos, o que sinaliza a importância de um maior apoio aos estudantes da área de saúde quanto maior sua faixa etária.

Rimmer (1982) relata que a estimativa na América do Norte de suicídios que iniciaram com quadros depressivos nos estudantes universitários, é de 10\%, principalmente nos dois últimos anos do curso. No Brasil, observou que a taxa de suicídio em acadêmicos foi, aproximadamente, quatro vezes maior do que a da população. Fato que motivou a criação do Grupo de Assistência Psicológica ao Aluno (GRAPAL) na faculdade de Medicina da Universidade de São Paulo (FMUSP) (AGUIAR, 2007).

Os resultados encontrados na presente pesquisa evidenciam a necessidade de criar um mundo solidário no cotidiano do meio acadêmico, com participação tanto dos coordenadores, quanto dos estudantes e familiares, promovendo ações específicas na identificação dos problemas psicológicos e emocionais. Devem-se detectar os sintomas depressivos e intervir com medidas de apoio, programas educacionais, estratégias clínicas para orientação e o diagnóstico precoce em tempo hábil, minimizando prejuízos para o futuro desses profissionais da área de saúde.

\section{CONCLUSÃO}

A presença de depressão foi observada nos três cursos pesquisados da área de saúde, sendo significativamente maior nos acadêmicos de Enfermagem, depois nos de Odontologia e com menor incidência nos de Medicina. O gênero feminino apresentou maior prevalência e o índice de depressão foi maior quanto maior a faixa etária, independente do curso avaliado.

\section{REFERÊNCIAS}

\section{AGUIAR, S. M. Prevalência de sintomas de estresse e de depressão nos estudantes de medicina e de odontologia, 2007. p.126.}

BECK, A.T.; STEER, R. A.; CARBIN, M. G. Psychometric properties of the Beck Depression Inventory: Twentyfive years of evaluation. Clin Psychol Rev., 8(1):77100, 1988.

COSTA, E. F. de O. et al. Sintomas depressivos entre internos de medicina em uma universidade pública brasileira. Rev Assoc Med Bras. Associação Médica Brasileira, 58(1):53-59, 2012.

COUTINHO, M. da P. de L. et al. Depression, a misery without border: social representations between children and elderly. Psico-USF. Universidade São Francisco, 8(2):183-192, 2003.

CUNHA, J. A. Manual da versão em português das escalas Beck. São Paulo: Casa do psicólogo, 2011. p.172. 
GOMES-OLIVEIRA, M. H. et al. Validation of the Brazilian Portuguese Version of the Beck Depression Inventory-II in a community sample. Rev Bras

Psiquiatr. Associação Brasileira de Psiquiatria (ABP), 34(4):389-394, 2012.

\section{FIORANO, A. M. M. 0 impacto das emoções no processo ensino-aprendizagem no curso de graduação em Enfermagem. São Paulo: Pontifícia Universidade Católica de São Paulo, 2002.}

FUREGATO, A. R. F. et al. Depression and self-esteem among nursing students. Rev Psiquiatr Clínica. São Paulo: Faculdade de Medicina da Universidade de São Paulo, 33(5):239-244, 2006.

HAGHPARAST, N. et al. Evaluation of student and faculty perceptions of the PBL curriculum at two dental schools from a student perspective: a crosssectional survey. Eur J Dent Educ., 11(1):14-22, 2007.

KAWAKAME, P. M. G.; GARCIA, T. M. Desvendando o significado de experiências clínicas iniciais de estudantes de graduação em enfermagem. Rev Bras Enferm. Associação Brasileira de Enfermagem, 53(3):355-362, 2000.

LIMA, D. Depressão e doença bipolar na infância e adolescência. J Pediatr, Rio de Janeiro: Sociedade Brasileira de Pediatria, 80(2):11-20, 2004.

LIMA, M. C. P.; DOMINGUES, M. de S; CERQUEIRA, A. T. de A. R. Prevalência e fatores de risco para transtornos mentais comuns entre estudantes de medicina. Rev Saude Publica. São Paulo: Faculdade de Saúde Pública da Universidade de São Paulo, 40(6):1035-1041, 2006.

MORO, A.; VALLE, J. B. do; LIMA, L. P. de. Sintomas depressivos nos estudantes de medicina da Universidade da Região de Joinville (SC). Rev bras educ méd., 29(2):97-102, 2005.
MOORE, G. T. Initiating problem-based learning at Harvard Medical School. In: BOUD, D. The challenge of problem based learning. Second. New York: Kogan, 1997.

\section{MOSCOVICI, S. Representações sociais:}

Investigações em Psicologia Social. Rio de Janeiro: Vozes, 2003

NARDI, A. E. Depressão no Ciclo da Vida. Rev Bras

Psiquiatr. Associação Brasileira de Psiquiatria (ABP), 22(3):151-152, 2000.

NEWBURY-BIRCH, D.; LOWRY, R. J.; KAMALI, F. The changing patterns of drinking, illicit drug use, stress, anxiety and depression in dental students in a UK dental school: a longitudinal study. Br Dent J., 192(11):646-649, 2002.

OHL RIB. Os sentimentos dos alunos de graduação da área da saúde na primeira experiência formal com o paciente hospitalizado. São Paulo: Escola Paulista de Medicina, 1991.

RIMMER, J.; HALIKAS, J. A.; SCHUCKIT, M. A. Prevalence and incidence of psychiatric illness in college students: a four year prospective study. J Am Coll Health., 30(5):207, 1982.

SANTOS, T. M. dos; ALMEIDA, A. O. de; MARTINS, H. O.; MORENO, V. Aplicação de um instrumento de avaliação do grau de depressão em universitários do interior paulista durante a graduação em Enfermagem. Acta Sci Heal Sci., 25(2):171-176, 2003.

SANTOS, T. M. dos; ALMEIDA, A. O. de; MARTINS, H. O.; MORENO, V. Aplicação de um instrumento de avaliação do grau de depressão em universitários do interior paulista durante a graduação em Enfermagem. Acta Sci Heal Sci. 25(2):171-176, 2003. 
1. Graduada em Enfermagem pela Universidade Estadual de Ciências da Saúde de Alagoas. Email: paulabarros-@hotmail.com

2. Graduada em Enfermagem pela Universidade Estadual de Ciências da Saúde de Alagoas. Email: carlinha gomes7@hotmail.com

3. Docente em Enfermagem da Universidade Estadual de Ciências da Saúde de Avaliado em: 21 de Julho de 2015 Alagoas e Mestre em Ciências pela Universidade Federal de São Paulo - UNIAceito em: 11 de Agosto de 2015 